\section{An Innovative Method for Imaging and Chemical Analysis of Wet Samples in Scanning Electron Microscopes}

Irit Ruach-Nir

QuantomiX Ltd, Rehovot, Israel

iritrn@quantomix.com

\section{Introduction}

Electron microscopy (EM) of fully wet samples is a valuable tool for studies in the material, medical and biological sciences. In order to appreciate the natural structures of tissues or materials they should be examined in their native wet state, as opposed to a dry form that incorporates artifacts of sample processing. Viewing and analyzing wet samples at high resolution has undergone a significant improvement only recently due to the innovative WETSEMTM technology developed by QuantomiX.[1,2] a

\section{QX-102}
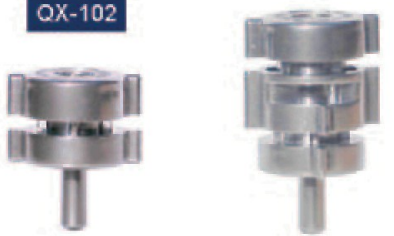

b

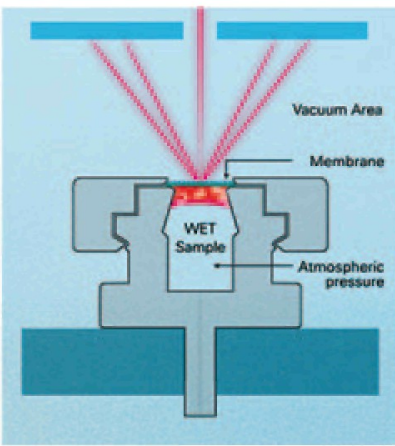

Figure 1 WETSEM ${ }^{\infty}$ technology: Fig Ia shows pictures of the Quantomix capsules $\mathrm{QX}-102$ and $\mathrm{QX}-302$. Fig 16 is a schematic representation of WETSEM ${ }^{\mathrm{n} x}$.

QuantomiX' capsules for WETSEMTM technology rely on a thin electron-transparent membrane that enables separation, and thereby protection, of the wet sample from the electron microscope vacuum (Figure 1a). Imaging is performed using a standard Scanning Electron Microscope (SEM) combined with a Back Scattered Electron (BSE) detector (Figure 1b). Our investigations into applications of WETSEMTM have highlighted some general advantages of using this new technology beyond its most obvious benefit: the revolutionary capability to image wet samples in an EM. A significant advantage of WETSEMTM over other electron microscopy techniques is the ability to image samples a few millimeters thick without any time-consuming, costly processing such as thin sectioning, embedding, freezing or coating. Our method of imaging is based on detection of BSEs, which result only from interactions of the electron beam with a thin membrane-proximal layer. Accordingly, any material beyond the layer of beam penetration is effectively invisible. The use of a BSE detector results in an image based on material contrast. Image contrast can sometimes be enhanced by staining samples with electron-dense stains. In summary, WETSEMTM combines the simple, rapid sample preparation of light microscopy with the high resolution capacity of EMs. It provides an ideal solution for immediate high resolution imaging of wet samples without drying artifacts. In this article we summarize recent developments and applications of this novel technology relevant to the material sciences.

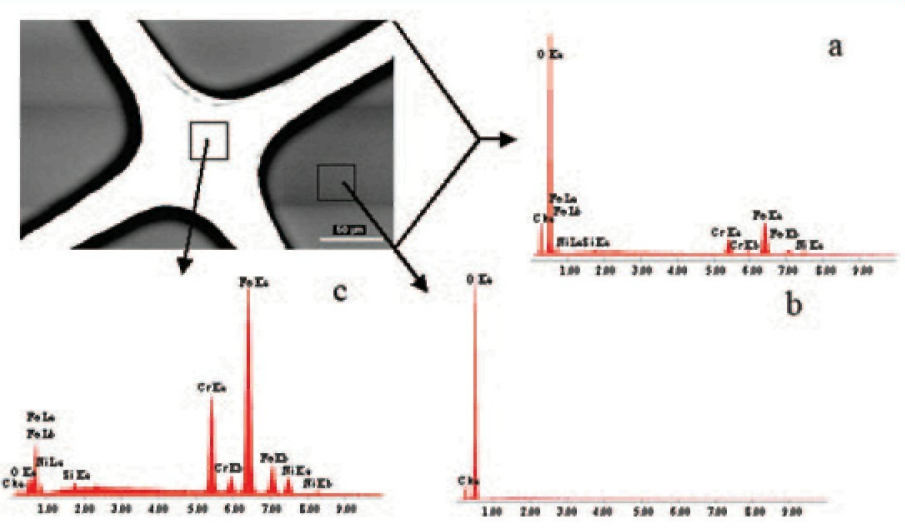

Figure 2 EDS using WETSEM ${ }^{\mathrm{m}}$ : Image of a capsule filled with double distilled water. EDS of image full-frame (a). EDS of area outside grid (b). EDS of area within grid (c). Bar: $50 \mu \mathrm{m}$

\section{Energy Dispersive X-Ray Spectroscopy (EDS) of Wet Samples}

Characterization of chemical microstructures is an important application of SEM. However, before the development of WETSEMTM technology this technique could not be applied to samples that are fully wet and under atmospheric pressure. We demonstrate here qualitative elemental microanalysis of samples in their native, fully wet state.

A sample is placed in a sealed QX capsule and analyzed using a conventional SEM equipped with an energy dispersive $\mathrm{X}$-ray spectrometer. In Figure 2 water was placed inside the QX
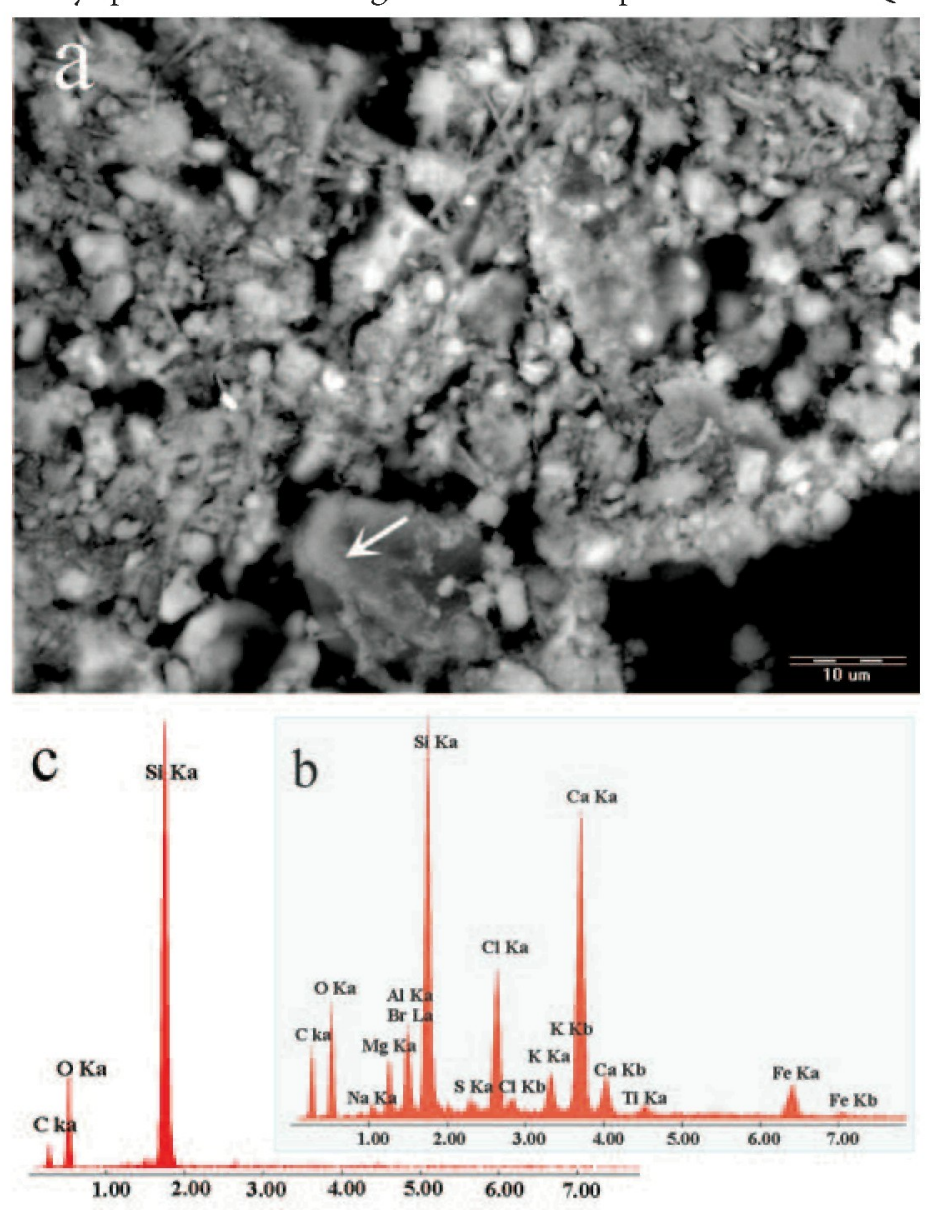

Figure 3 WETSEM $^{\text {тм }}$ image and EDS of Dead Sea mud: Image of Dead Sea mud (a). EDS spectrum of image full-frame (b). EDS of area marked by arrow in image $3 a$ (c).Bar: $10 \mu \mathrm{m}$ 


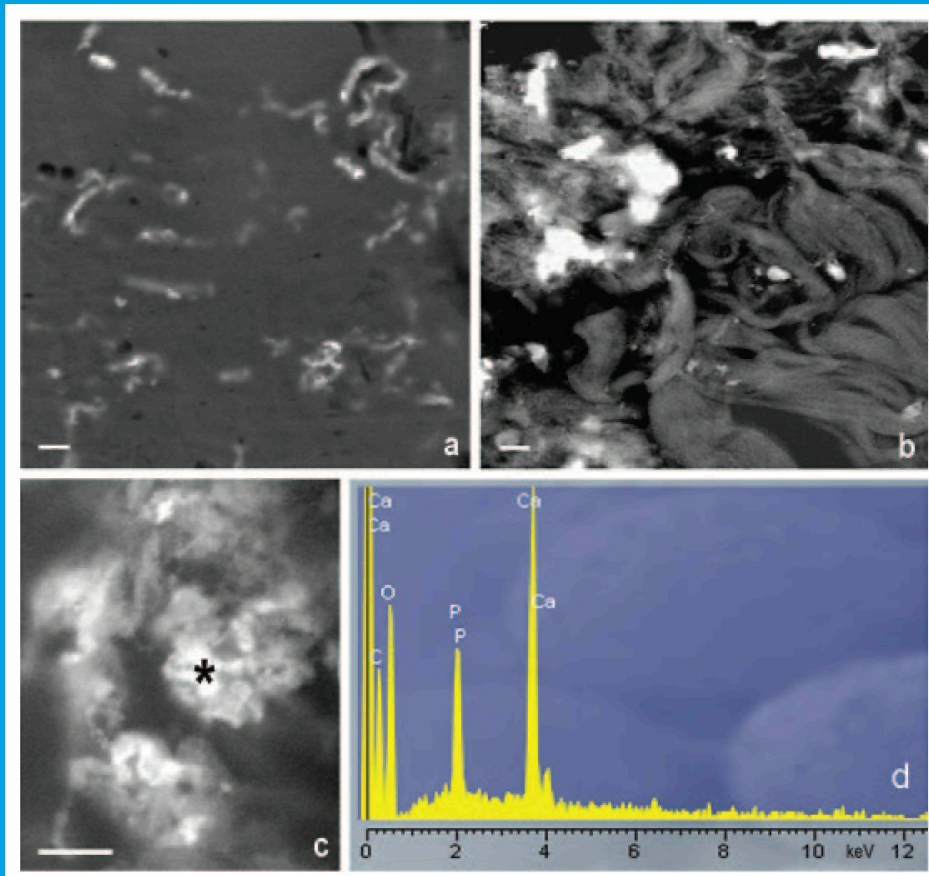

Figure 4 WETSEM images of PXE dermal biopsies (bar: $10 \mathrm{um}$ ): Skin biopsy unfixed and unstained (a), fixed and stained with uranyl acetate (b), high magnification image of mineralized elastin (c) and microanalysis (d) of mineralized area of elastin (denoted by ${ }^{*}$ in $4 c$ ). The biopsies were placed in the QX capsule and observed and analyzed by conventional SEM equipped with an EDS detector. (Images and EDS spectrum courtesy of Daniela Quaglino et al.).

capsule and analyzed by EDS. The oxygen characteristic peak is readily observed (Figure $2 \mathrm{~b}$ ). The contribution to the EDS spectrum of the thin membrane is negligible, a small carbon peak that does not interfere with microanalysis (Figure $2 b$ ). The metal grid (bright cross in the image) does generate a large EDS signal (Figure $2 \mathrm{c}$ ). When the analyzed area contains part of the grid, the spectrum will include the grid signature (Figure $2 a)$ as well as the oxygen and carbon peaks. However, when the analyzed area does not contain the grid, it will not contribute to the elemental signature (Figure $2 \mathrm{~b}$ ).

In Figure 3 we present a WETSEMTM image (Figure 3a) and an EDS spectra of a sample of Dead Sea mud (courtesy of AHAVA cosmetic laboratories). In figure $3 \mathrm{~b}$ the elemental signature from the area seen in the image is shown ('full-frame' analysis). In figure $3 c$ we demonstrate 'spot' analysis within that area (see arrow in figure $3 \mathrm{a}$ ) that identifies silicon as the major constituent at that position. When using EDS the natural heterogeneity of the sample is retained and adjacent structures can be directly compared by spot analysis.

The ability to carry out EDS of wet samples has excited the interest of biologists as well as material scientists. Pseudoxanthoma elasticum (PXE) is a genetic disorder affecting the skin, eye and cardiovascular system. The majority of disease alterations can be attributed to degeneration of elastic fibers that undergo progressive mineralization and fragmentation. To date, costly and time-consuming light microscopy and EM analyses of skin biopsies, that require large amounts of material, are the means to identify mineral precipitates and diagnose PXE. A new study has demonstrated that WETSEMTM technology enables rapid, accurate localization and detection of mineralized areas in unfixed and unstained as well as fixed and stained small PXE biopsy specimens (Figure 4).[3] The mineralized areas are easily observed in images of both unstained and stained samples (Figures $4 \mathrm{a}$ and $4 \mathrm{~b}$ ). Since the mineralized areas are rich in elements with higher electron density than the rest of the tissue, they appear brighter. Moreover, EDS allowed delineation of the elemental composition of the mineralized areas of elastin (Figures $4 \mathrm{c}$ and $4 \mathrm{~d}$; note the EDS characteristic peaks of $\mathrm{Ca}$ and $\mathrm{P}$ ) and even the relative ratio of element's deposits.[3] Thus, WETSEMTM may be the means to rapidly and economically diagnose PXE and perhaps other tissue disorders that involve ion precipitation. It is notable that these experiments employed a newly developed QX capsule (QX-302). This capsule is designed to facilitate WETSEMTM of tissues and other soft wet samples. It has a positioning element to place samples next to the membrane (Figure 1A)

\section{WETSEM $^{\mathrm{TM}}$ of Native Material Samples}

Before the advent of WETSEMTM it was not possible to use EM to image particles in suspension. The samples had to be dried in order to be imaged. However, after drying much of the information on the distribution of particles in the solution was lost since aggregation often occurred during the drying process. The two images of Figure 5 illustrate the different structures seen by SEM depending on the hydration status of a sample. The images show Ag nanoparticles that were synthesized by reduction of silver ions in water and stabilized with a negatively charged cupping agent. The wet sample was prepared by inserting $15 \mu \mathrm{l}$ of the suspension into a QX-102 capsule with a poly-l-lysine layer coating the membrane. The poly-1-lysine (positively charged polymer) layer is essential in order to assure close proximity of the particles to the membrane. The dry sample was prepared by drying a drop of the Ag nanoparticle suspension on a SEM stub covered with a carbon tape. It is clear that little information on the degree of aggregation or isolation of the particles in the wet environment (Figure 5a) can be gained from the image of the dry sample (Figure 5b).
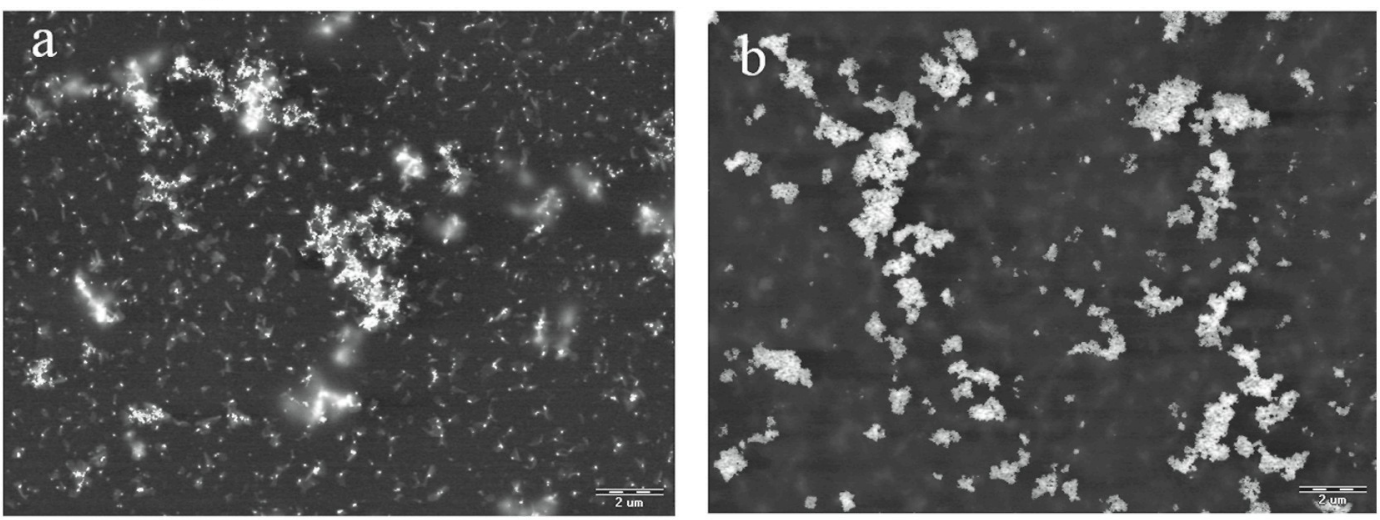

Figure 5 WETSEM ${ }^{\mathrm{ix}}$ of Ag nanoparticles: Wet sample (a). Dry sample (b). 


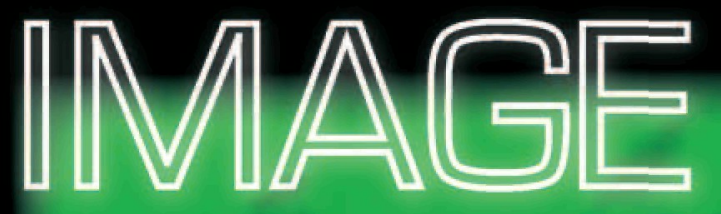

THE POSSIBILITIES
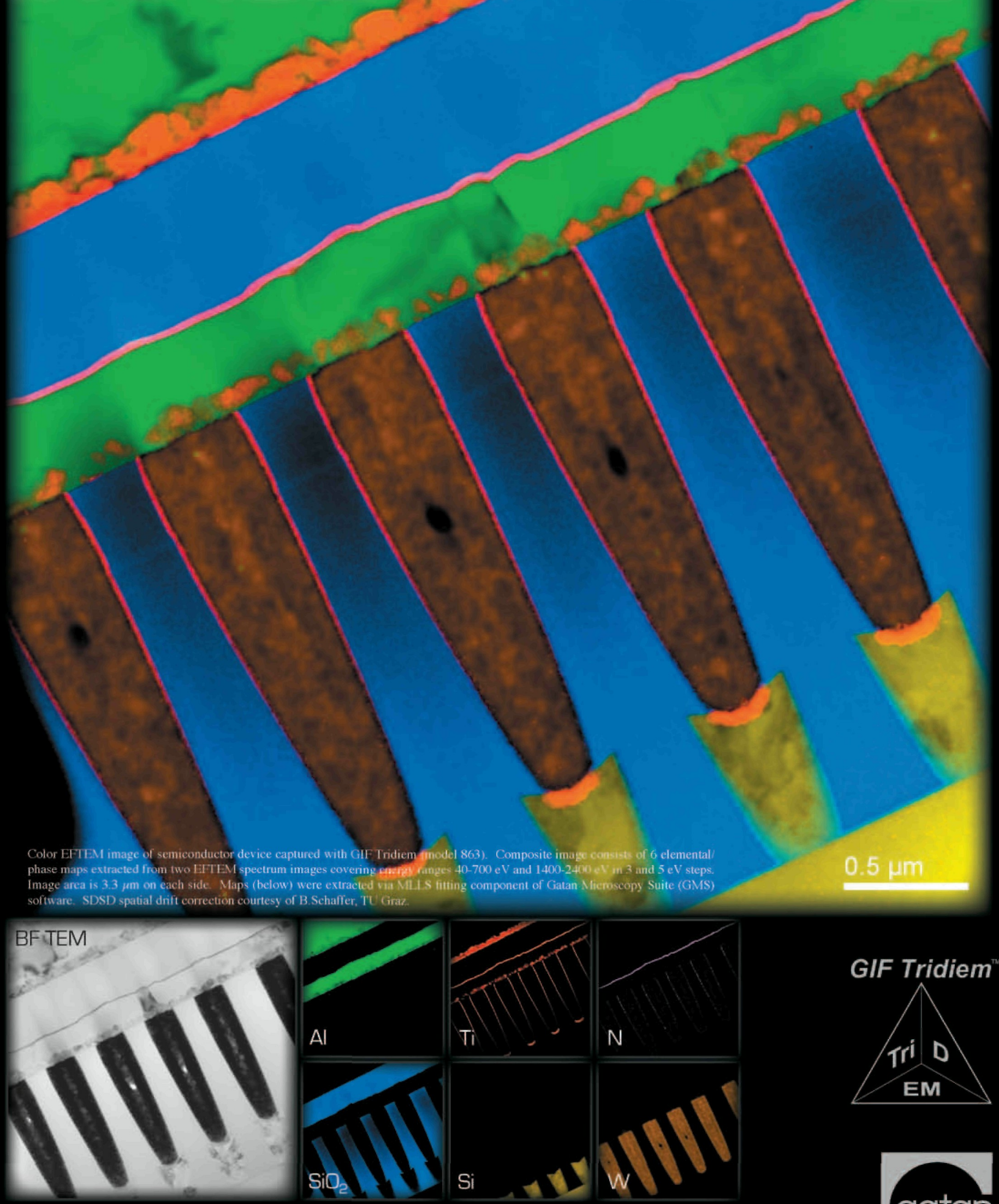

$\mathrm{siO}_{2} \mid 1$

Visit us at

Microscopy \& Microanalysis 2005 in booth 1007 . 


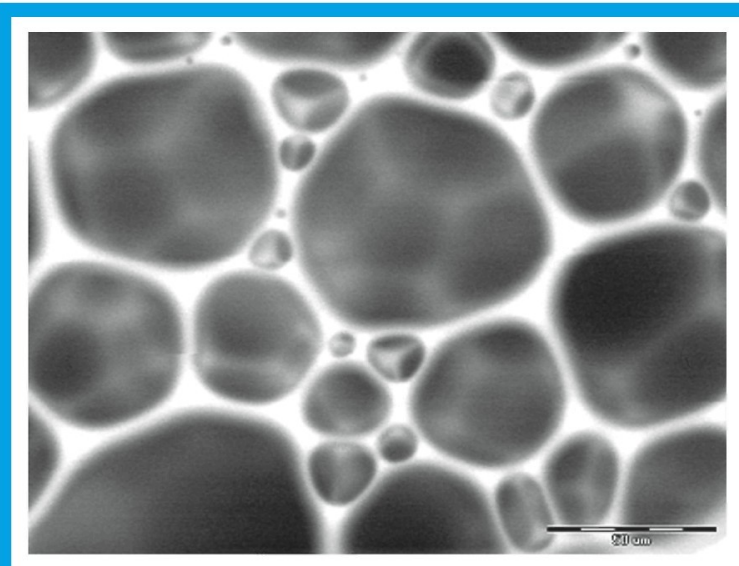

Figure 6: WETSEM ${ }^{\mathrm{mx}}$ of Shaving Foam
Examples of wet, or partially wet samples, or samples with unusual consistency that could not be imaged by SEM until now include shaving foam (Figure 6) and deodorant (Figure 7), a volatile material. WETSEMTM technology represents a potentially useful tool for formulation and control processes in commercial and industrial fields. A good example where WETSEMTM technology was used to monitor product quality is illustrated by the images in Figure 8. A sample of pesticide was viewed at lower (Figure $8 \mathrm{a}$ ) and higher magnification (Figure $8 \mathrm{~b}$ ) to assess the proportion of pesticide contained within capsules designed for controlled release. The two phases can be distinguished easily and it appears that the majority of the active material (gray in the image) is not encapsulated, indicating the need to improve the formulation.

In summary, the innovative WETSEMTM technology facilitates economic, simple, rapid, high resolution imaging and EDS analysis of fully wet samples in a way that has not been possible before. Potential applications for the material sciences are only

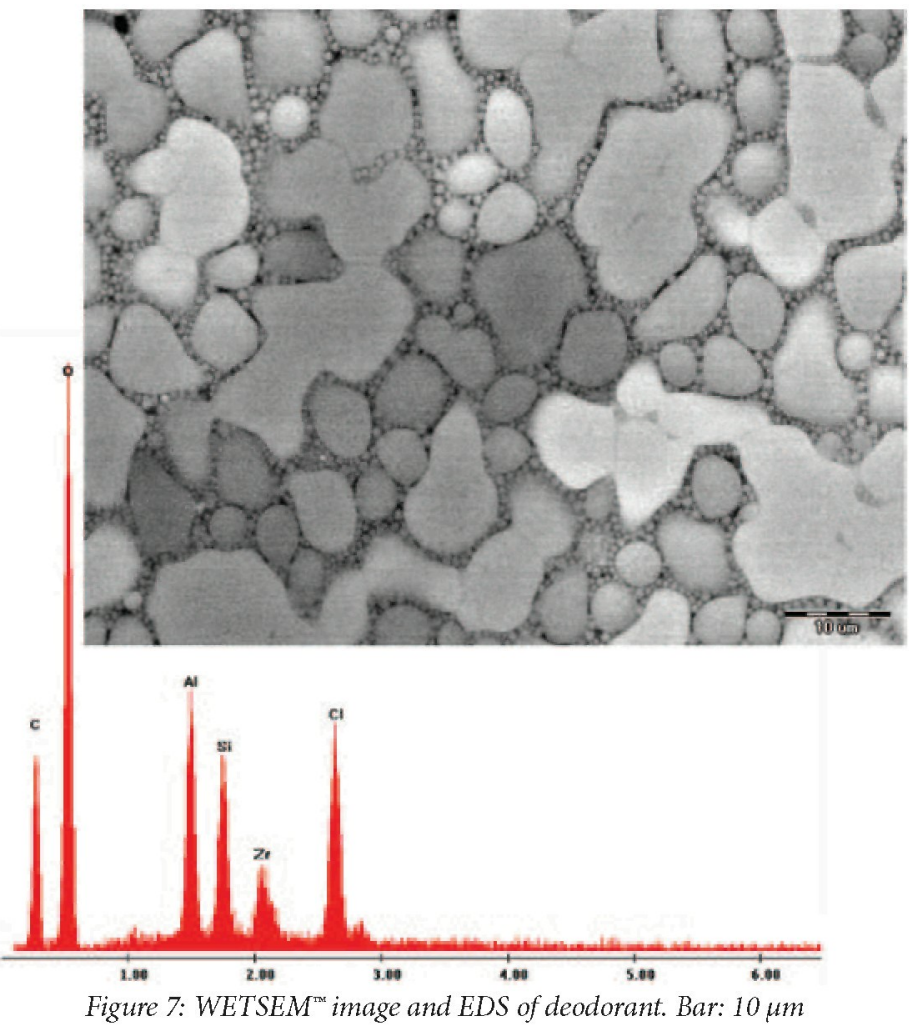

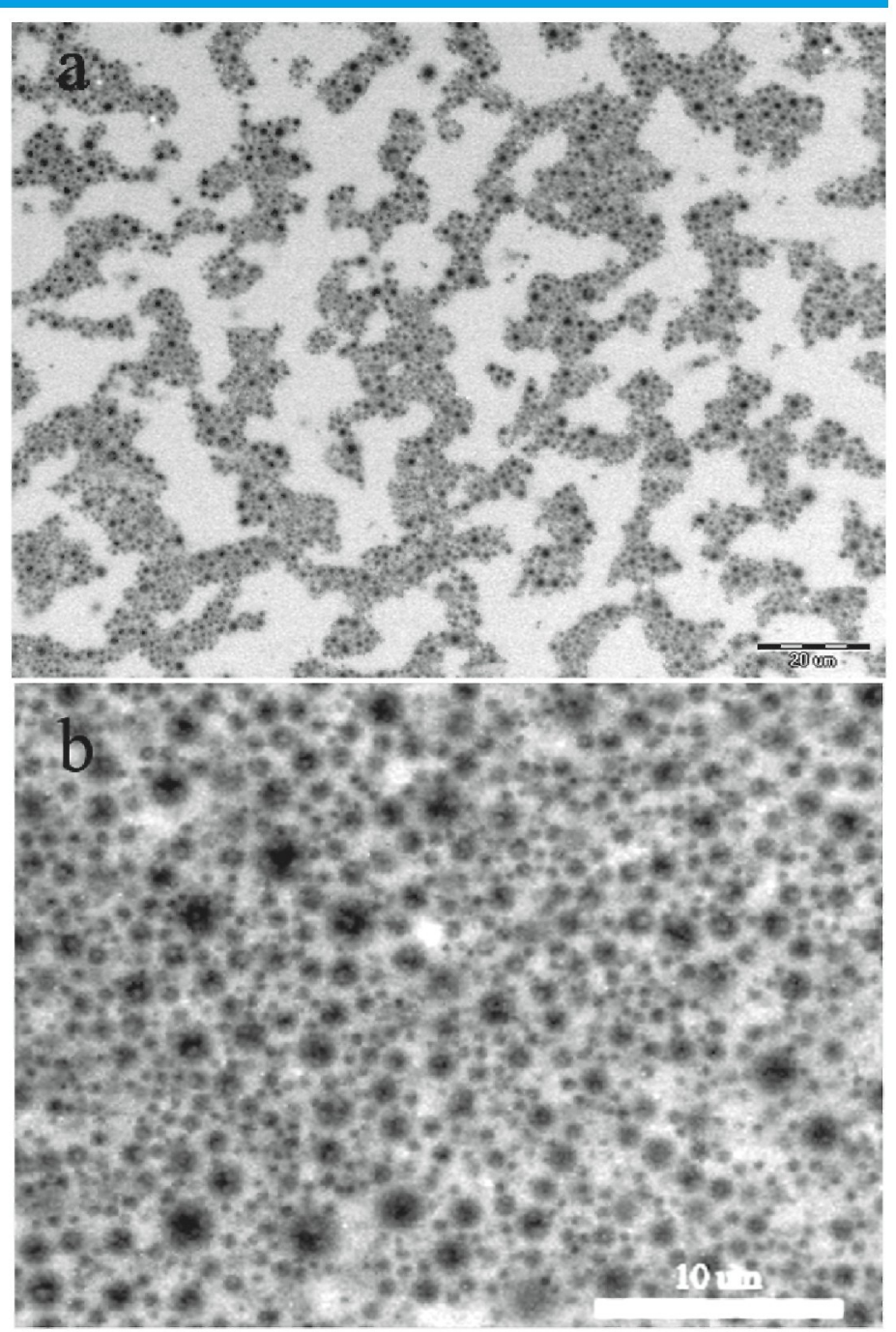

Figure 8 WETSEM ${ }^{\mathrm{mx}}$ of pesticide: Lower (a) and higher (b) magnifications

just beginning to be realized and tested.

\section{Acknowledgements:}

We would like to thank Prof. Shlomo Magdassi for providing us the silver nanoparticles and for our fruitful discussions. We would also like to thank Prof. Daniela Quaglino for the permission to use her results on wet mineralized samples. We thank AHAVA cosmetic laboratories for the Dead Sea mud samples.

\section{References:}

1. S. Thieberge, O. Zik, E. Moses. An apparatus for imaging liquids, cells and other wet samples in the scanning electron microscope, (2004) Rev. Sci. Instruments, 75, 2280-2289.

2. S. Thieberge, A. Nechushtan, D. Shprinzak, O. Gileadi, V. Behar, O. Zik, Y. Chowers, S. Michaeli, J. Schlessinger, E. Moses. Scanning electron microscopy of cells and tissues under fully hydrated conditions, (2004) Proc. Natl. Acad. Sci. USA 101, 3346-3351.

3. F. Boraldi, M. Tonelli, D. Gheduzzi, I. Pasquali Ronchetti and D. Quaglino, Identification of mineralized elastic fibers on wet samples by SEM, Microscopy Research and Technique, in press. 


\section{Journal of}

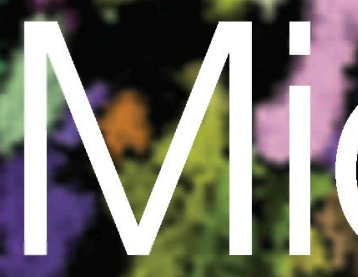

A leading journalpublishing the most rajoily expanding techniquesfin science

10 good reasons to submit your paper to Journal of Microscopy

- No page charges

- No submission fee

- No colour charges

- No page limit

- Submission to first decision within 55 days

- Rapid publication time

- Author Services - track your article from acceptance to print

- Online submission at http://jmi.manuscriptcentral.com/

- High readership figures

- Widely Abstracted and Indexed

Visit the journal online at

www.journalofmicroscopy.com

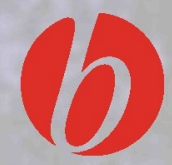

Blackwell Publishing 